\title{
Some applications of Burzyński yield condition in metal plasticity
}

\author{
G. Vadillo ${ }^{\mathrm{a}, *}$, J. Fernández-Sáez $^{\mathrm{a}}$, R.B. Pęcherski ${ }^{\mathrm{b}}$ \\ ${ }^{a}$ Department of Continuum Mechanics and Structural Analysis, University Carlos III of Madrid, Avda. de la Universidad 30, 28911 Leganes, Madrid, Spain \\ ${ }^{\mathrm{b}}$ Department of Mechanics of Materials, Institute of Fundamental Technological Research, Polish Academy of Sciences, Pawinskiego 5B, 02-106 Warsaw, Poland
}

\section{Keywords:}

A. Metal matrix

E. Mechanical

F. Plastic behaviour

\begin{abstract}
A B S T R A C T
The classical $J_{2}$ plasticity theory is widely used to describe the plastic response of metallic materials. However, this theory does not provide satisfactory predictions for materials which exhibit pressure sen sitive yielding or plastic dilatancy. Another difficulty is the difference between the values of yield stresses in tension and compression for isotropic materials, the so called strength differential effect (SD), leading to the asymmetry of the elastic range. The Burzyński yield condition, proposed in 1928, can be used to overcome some of these problems. In this paper an implicit integration of the elasto plastic constitutive equations for the paraboloid case of Burzyński's yield condition is formulated. Also, the tangent operator consistent with the integration algorithm was developed and is presented. The proposed model was implemented in a commercial Finite Element code and different kinds of tests reported in the literature were simulated. The comparison between the numerical and experimental results shows that the plastic ity theory with the paraboloid case of Burzyński's yield condition describes adequately the strength dif ferential effect, which is present in many kinds of materials significant for recent applications.
\end{abstract}

\section{Introduction}

The analysis of complex structural components, their design optimization and structural reliability assessment require the use of proper and accurate constitutive models to describe a material behaviour. To date, an overwhelming majority of structural analy sis employ the classical $J_{2}$ plasticity theory to describe the plastic response of metallic alloys. This theory assumes that hydrostatic stress has no effect on plastic flow and the material is incompress ible in the plastic regime.

However, there are reported in the literature on experimental results for metallic solids under quasi static conditions, which ex hibit pressure sensitive yielding and plastic dilatancy and reveal inconsistency of the model based on the Huber Mises yield condi tion $[1,2]$. In particular, this effect can be significant in designing, structural elements or machine parts in which stress concentra tions may appear resulting in the increase of the value of the first stress invariant.

Another difficulty to be taken into account is the difference of the values of yield stresses in tension and compression for isotropic materials, the so called strength differential effect, leading to the asymmetry of the elastic range.

The observed hydrostatic stress effect on the yield behaviour of the investigated metallic materials is commonly described in the literature by means of the adaptation of the criterion known in

\footnotetext{
* Corresponding author. Fax: +34 916248809.

E-mail address: gvadillo@ing.uc3m.es (G. Vadillo).
}

the soil mechanics in which the linear dependence of the yield lim it on hydrostatic stress is assumed. Such a criterion was proposed originally in 1928 by Burzyński [3] and repeated later by other authors, e.g. by Drucker and Prager [4,5]. Recently, this kind of cri terion has been used to model the pressure sensitive yielding of metals [6 8]. However, it is known that the criterion represented by a conical failure surface in the space of principal stresses can only roughly approximate real behaviour of a material in the lim ited range of hydrostatic stress and fails to describe properly the states near to the apex of the failure cone [9].

In this paper the general Burzyński yield condition is reviewed showing that the Huber Mises, Drucker Prager and paraboloid Burzyński Torre conditions can be received as particular cases of this more general model.

Then, an implicit integration of the elastoplastic constitutive equations for the paraboloid Burzyński yield condition is formu lated. Also, the tangent operator consistent with the integration algorithm was developed and is presented. The proposed model was implemented in the Finite Element code ABAQUS through the user subroutine UMAT. This is a new contribution, which can find wide applications in practical analysis of complex states of stress in plasticity as well as viscoplasticity of metals and metal matrix composites [10]. In the latter paper an identical paraboloid yield criterion was considered. The authors related it with the names of Mises and Schleicher ([11] for a more detailed discussion of the historical background of the development of the paraboloid failure criteria). However, Zhang et al. [10] do not provide an inte gration algorithm of the developed equations of plasticity with the 
paraboloid yield condition or an implementation into a finite ele ment code. Therefore, the results presented in our paper are impor tant and relevant for recent applications.

In order to validate the discussed constitutive model of plas ticity with the paraboloid case of Burzyński's yield condition, the tensile test on notched tensile specimens of the $2024 \mathrm{~T} 3$ alumi num alloy performed earlier by Wilson [6] were simulated. The computations were compared with the experimental data and the results of numerical calculations made with the application of the Drucker Prager yield condition presented in [6]. Also, the experimental data obtained by Iyer and Lissenden [12] for the polycrystalline nickel base alloy Inconel 718 , which reveals the strength differential effect, were applied. Numerical simula tions of experimental tests of a thin walled tube subjected to tension combined with torsion, as well as a thin walled tube subjected to compression combined with torsion, were per formed. Such non proportional loading paths containing corners provide a rigorous test of a plasticity model. The comparison of the results of computations with experimental data shows that the application of the plasticity theory with the Burzyński case of the paraboloid yield condition is more consistent with experiment than the classical plasticity theory with the Huber Mises yield condition.

\section{The Burzyński yield condition}

The study of the literature of the subject shows that the ori ginal results of Burzyński presented in his doctoral thesis and in his further papers $[3,13]$ are of fundamental significance and re main important also for recent studies of models of solids with asymmetric behaviour of the elastic range. It concerns soils and rocks (e.g. applications in modelling of interaction of a cut ting tool with geological settings [14]), as well as modern mate rials (e.g. polymers [15], composites and porous solids [16,17].

The concept of the Burzyński yield condition was presented in detail and compared with several later independent propositions by Życzkowski $[18,19]$ as well as Skrzypek [20] and Jirásek et al. [21]. The yield criterion proposed by Huber, Mises and Hencky [22 24] for isotropic solids characterized with equal values of yield stresses in tension and compression was well established and con firmed experimentally in the twenties of the last century. The open question remained, however, in the subject of yield criteria for isotropic materials revealing different values of yield stresses in tension and compression, the so called strength differential (SD) effect.

Yield conditions with plastic behaviour depending on hydro static pressure may be described by equations of rotationally symmetric surfaces in the space of principal stresses with the sym metry axis: $\sigma_{1}=\sigma_{2}=\sigma_{3}$. Their general form is: $f\left(\sigma_{m}, \sigma_{e}\right)=0$ ) where $\sigma_{m}$ is the hydrostatic stress and $\sigma_{e}$ corresponds to the effective stress.

$\sigma_{m} \quad \frac{1}{3} \boldsymbol{\sigma}: \mathbf{1}, \quad \sigma_{e} \quad \sqrt{\frac{3}{2}} \boldsymbol{\sigma}^{\prime}: \boldsymbol{\sigma}^{\prime}, \quad \boldsymbol{\sigma}^{\prime} \quad \boldsymbol{\sigma} \quad \sigma_{m}: \mathbf{1}$

with $\boldsymbol{\sigma}$ the Cauchy stress tensor, $\boldsymbol{\sigma}^{\prime}$ the stress deviator, and $\mathbf{1}$ the unit second order tensor.

The Burzyński's formulation in the general form $f\left(\sigma_{m}, \sigma_{e}\right)=0$ is presented as a three parameter condition, having physical founda tions in an energy based criterion $[3,11,13]$ :

$A \sigma_{e}^{2}+B \sigma_{m}^{2}+C \sigma_{m} \quad 1 \quad 0$

Burzyński evaluated the parameters $A, B$ and $C$ using uniaxial ten sion (to obtain the yield stress $\sigma_{Y}^{T}$ ), uniaxial compression (to obtain $\left.\sigma_{Y}^{C}\right)$ and simple shear tests $\left(\tau^{S}\right)$ resulting in the following [19]:
$\frac{\sigma_{Y}^{T} \sigma_{Y}^{C}}{\left(3 \tau^{S}\right)^{2}} \sigma_{e}^{2}+\left(9 \quad \frac{3 \sigma_{Y}^{T} \sigma_{Y}^{C}}{\left(\tau^{S}\right)^{2}}\right) \sigma_{m}^{2}+3\left(\begin{array}{cccc}\sigma_{Y}^{C} & \left.\sigma_{Y}^{T}\right) \sigma_{m} & \sigma_{Y}^{C} \sigma_{Y}^{T} & 0\end{array}\right.$

In order to account for isotropic hardening $\sigma_{Y}^{T}, \sigma_{Y}^{C}$ and $\tau^{S}$ are as sumed to be dependent on the effective plastic deformation $\bar{\varepsilon}^{p}$. This equation describes in the space of principal stresses a hyperboloid (if $3\left(\tau^{S}\right)^{2}<\sigma_{Y}^{C} \sigma_{Y}^{T}$ ), or an ellipsoid (if $3\left(\tau^{S}\right)^{2}>\sigma_{Y}^{C} \sigma_{Y}^{T}$ ). To reduce the number of independent parameters to two, some particular cases of the relation between $\tau^{S} \sqrt{ } 3$ with $\sigma_{Y}^{T}$ and $\sigma_{Y}^{C}$ are analysed [19].

With the relation:

$\tau^{S} \sqrt{ } 3 \quad \frac{2 \sigma_{Y}^{T} \sigma_{Y}^{C}}{\sigma_{Y}^{T}+\sigma_{Y}^{C}}$

Eq. (3) turns into the formula of a circular cone (Burzyński Druc ker Prager cone):

$\sigma_{e}+3 \frac{\sigma_{Y}^{C} \quad \sigma_{Y}^{T}}{\sigma_{Y}^{C}+\sigma_{Y}^{T}} \sigma_{m} \quad 2 \frac{\sigma_{Y}^{C} \sigma_{Y}^{T}}{\sigma_{Y}^{C}+\sigma_{Y}^{T}} \quad 0$

while with $\tau^{s} \sqrt{ } 3 \quad \sqrt{ } \sigma_{Y}^{T} \sigma_{Y}^{C}$ the relation in Eq. (3) transforms into the equation of a paraboloid, called by Życzkowski the Burzyński Torre paraboloid yield condition $[19,25]$.

$\sigma_{e}^{2}+3\left(\sigma_{Y}^{C} \quad \sigma_{Y}^{T}\right) \sigma_{m} \quad \sigma_{Y}^{C} \sigma_{Y}^{T} \quad 0$

Introducing the relation between uniaxial compression and uniaxial tension yield stresses $k \quad \sigma_{Y}^{C} / \sigma_{Y}^{T}$, Eq. (6) can be written as:

$\sigma_{e}^{2}+3(k \quad 1) \sigma_{m} \sigma_{Y}^{T} \quad\left(\sigma_{Y}^{T}\right)^{2} k \quad 0$

after solving with respect to $\sigma_{Y}^{T}$ and extracting the positive root, this equation takes the form:

$\frac{1}{2 k}\left\{3\left(\begin{array}{ll}k & 1\end{array}\right) \sigma_{m}+\sqrt{9}\left(\begin{array}{ll}k & 1\end{array}\right)^{2} \sigma_{m}^{2}+4 k \sigma_{e}^{2}\right\} \quad \sigma_{Y}^{T} \quad 0$

Further, if $\sigma_{Y}^{C} \quad \sigma_{Y}^{T} \quad \sigma_{Y}$ and $\tau^{S} \quad \sigma_{Y} / \sqrt{ } 3$, the Huber Mises circular cylinder is obtained.

The relevant limit curves of the Burzyński model in the plane $\sigma_{m} \quad \sigma_{e}$ are shown in Fig. 1.

Unlike yield formulations such as the Huber Mises and Drucker Prager criteria or others, having mostly an empirical character, the Burzyński model has not been implemented into a commercial finite element code. In this article, the Burzyński paraboloid model is ana lysed and used for the first time in the algorithm of integration of the plasticity equations with the paraboloid yield condition and imple mented in the Finite Element program ABAQUS.

\section{Integration of the plastic equations with the Burzyński-Torre paraboloid yield condition}

Within the finite element method the integration process is local in space and occurs at quadrature points of the finite ele ments. The incremental integration of the constitutive model is a strain driven process in which the total strain tensor increment at each quadrature point is given at a certain time and both the stress and the state variables should be updated.

For structural metals elastic strains are usually very small com pared to unity or to plastic strains. For hypo elastic materials and with this restriction, the rate of deformation tensor $\dot{\boldsymbol{\varepsilon}}$, can be decomposed as the sum of an elastic $\dot{\boldsymbol{\varepsilon}}^{\boldsymbol{e}}$ and a plasic part $\dot{\boldsymbol{\varepsilon}}^{p}$ into the form:

$\dot{\varepsilon} \quad \dot{\varepsilon}^{e}+\dot{\varepsilon}^{p}$

and the stress rate, in terms of the elastic deformation rate tensor is:

$\dot{\boldsymbol{\sigma}} \quad \mathbf{C}:\left(\begin{array}{ll}\dot{\boldsymbol{\varepsilon}} & \dot{\boldsymbol{\varepsilon}}^{p}\end{array}\right)$ 


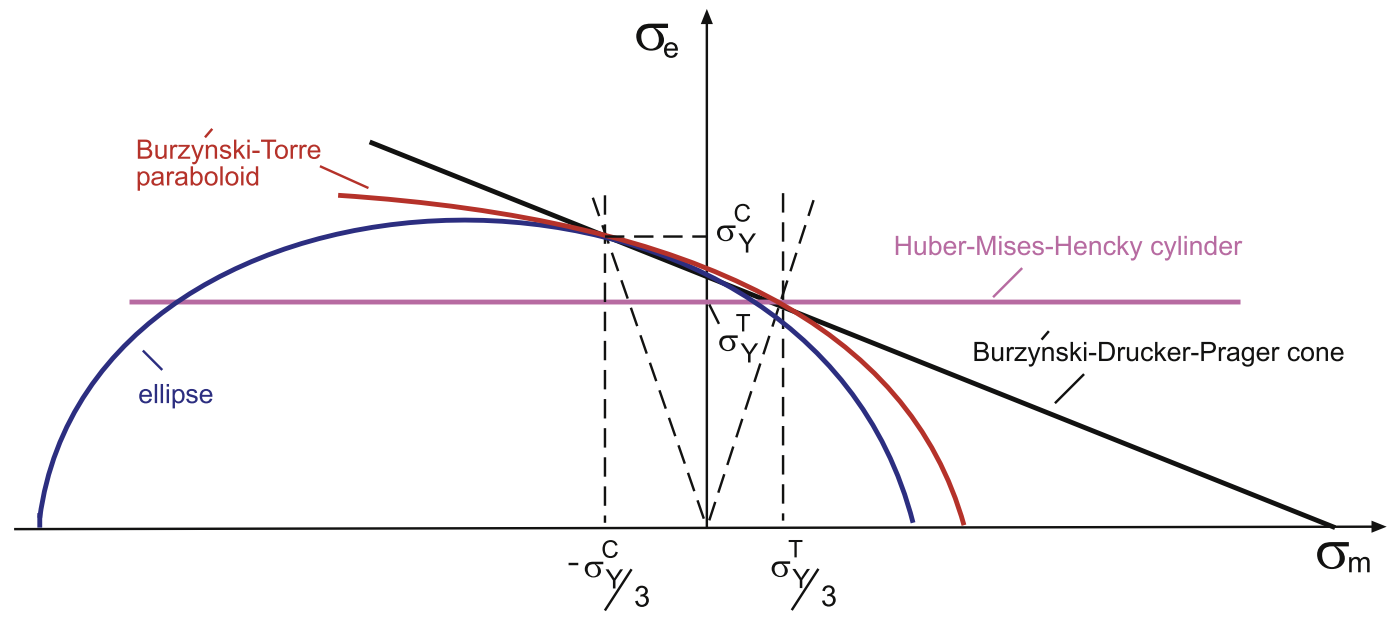

Fig. 1. The Burzyński yield condition and its specific cases: an ellipsoid, paraboloid, cylinder and cone.

In the above equation $\mathbf{C}$ is the tensor of isotropic elastic moduli:

$$
\text { C } 2 G \mathbf{I}^{\prime}+K \mathbf{1} \otimes \mathbf{1}
$$

where $G=E / 2(1+v)$ and $K=E / 3(1 \quad 2 v)$ are elastic constant, and $E$ and $v$ are Young's modulus and Poisson's ratio respectively. Also 1 is the unit second order tensor and $\mathbf{I}^{\prime}$ is the unit deviatoric fourth order tensor. The plastic part of the rate of deformation tensor has a direction normal to the flow potential, i.e:

$\dot{\boldsymbol{\varepsilon}}^{\boldsymbol{p}} \quad \dot{\lambda} \frac{\partial \Phi}{\partial \boldsymbol{\sigma}}$

with $\dot{\lambda}$ being the plastic proportionality factor, and $\Phi$ the Bur zyński Torre paraboloid yield surface:

$\Phi\left(\sigma_{m}, \sigma_{e}, \sigma_{Y}^{T}\right) \quad \frac{1}{2 k}\left\{3\left(\begin{array}{ll}k & 1\end{array}\right) \sigma_{m}+\sqrt{9}\left(\begin{array}{ll}k & 1\end{array}\right)^{2} \sigma_{m}^{2}+4 k \sigma_{e}^{2}\right\} \quad \sigma_{Y}^{T}$

The plastic part of the macroscopic strain increment and the effective plastic strain increment are related by:

$\boldsymbol{\sigma}: \dot{\boldsymbol{\varepsilon}}^{p} \quad \sigma_{Y}^{T} \dot{\varepsilon}^{p}$

The plastic flow proportionality factor $\dot{\lambda}$ can be written as:

$\dot{\lambda} \frac{\sigma_{Y}^{T} \dot{\varepsilon}^{p}}{\boldsymbol{\sigma}: \frac{\partial \Phi}{\partial \boldsymbol{\sigma}}}$

To complete the formulation, the Kuhn Tucker loading unload ing conditions are considered:

$\dot{\lambda} \geqslant 0, \quad \Phi \leqslant 0, \quad \dot{\lambda} \Phi \quad 0$

which means that $\dot{\lambda} \quad 0$ and $\Phi<0$ during elastic loading or unload ing and $\dot{\lambda}>0$ and $\Phi=0$ during plastic loading. The equation $\dot{\lambda} \Phi \quad 0$ represents the consistency condition.

To integrate the set of non linear equations into a finite element scheme at the level of a material point two different tasks must be accomplished. The first one consists in update stress and state vari ables driven by the strain increment. The second is related to cre ate a consistent tangent operator to preserve the quadratic convergence of the iterative solution based on Newton's method.

Numerical solutions of Eqs. (10), (12), (14), (16) in order to obtain $\dot{\boldsymbol{\sigma}}, \dot{\boldsymbol{\varepsilon}}^{p}, \dot{\lambda}$ and $\dot{\bar{\varepsilon}}^{p}$, are developed following the fully implicit backward Euler integration using the classical return mapping algorithm [26,27]. The return is performed at time $(n+1)$ with the corresponding updated stress:

$\boldsymbol{\sigma}_{(n+1)} \quad \boldsymbol{\sigma}_{(n+1)}^{\text {trial }} \quad \mathbf{C}: \Delta \boldsymbol{\varepsilon}^{p} \quad \boldsymbol{\sigma}_{(n+1)}^{\text {trial }} \quad\left(2 G \mathbf{I}^{\prime}+K \mathbf{1} \otimes \mathbf{1}\right): \Delta \boldsymbol{\varepsilon}^{p}$ with the trial stress given by:

$\boldsymbol{\sigma}_{(\boldsymbol{n}+1)}^{\text {trial }} \quad \boldsymbol{\sigma}_{(\boldsymbol{n})}+\mathbf{C}: \Delta \boldsymbol{\varepsilon}$ have:

From the yield condition during plastic loading in Eq. (16) we

$\Phi \quad 0$

From the associated flow rule in Eq. (12) and separating hydro static and deviatoric components we obtain:

$\Delta \boldsymbol{\varepsilon}^{\boldsymbol{p}} \quad \Delta \lambda\left(\frac{\partial \Phi}{\partial \boldsymbol{\sigma}}\right)_{(n+1)} \quad \Delta \lambda\left(\frac{1}{3} \frac{\partial \Phi}{\partial \sigma_{m}} \mathbf{1}+\frac{3}{2} \frac{\boldsymbol{\sigma}^{\prime}}{\sigma_{e}} \frac{\partial \Phi}{\partial \sigma_{e}}\right)_{(n+1)}$

or:

$\Delta \boldsymbol{\varepsilon}^{p} \quad \frac{1}{3} \Delta \varepsilon_{p} \mathbf{1}+\Delta \varepsilon_{q} \mathbf{n}_{(n+1)}$

In the above: $\mathbf{n}_{(n+1)}=\left(3 \boldsymbol{\sigma}^{\prime} /\left(2 \sigma_{e}\right)\right)_{(n+1)}$ is the unit vector in the devia toric space normal to the yield surface, and $\Delta \varepsilon_{p}$ and $\Delta \varepsilon_{q}$ are vari ables introduced by Aravas [28] in the following form:

$\Delta \varepsilon_{p} \quad \Delta \lambda\left(\frac{\partial \Phi}{\partial \sigma_{m}}\right)_{(n+1)}, \quad \Delta \varepsilon_{q} \quad \Delta \lambda\left(\frac{\partial \Phi}{\partial \sigma_{e}}\right)_{(n+1)}$

and transformed using Eq. (22) to eliminate $\Delta \lambda$ into:

$\Delta \varepsilon_{p}\left(\frac{\partial \Phi}{\partial \sigma_{e}}\right)_{(n+1)} \Delta \varepsilon_{q}\left(\frac{\partial \Phi}{\partial \sigma_{m}}\right)_{(n+1)} 0$

Bearing in mind the relation $\mathbf{n}_{(n+1)} \quad \mathbf{n}_{(n+1)}^{\text {trial }}$ [29], and introducing the expression of Eq. (21) in Eq. (18), it can be written that:

$\begin{array}{llll}\boldsymbol{\sigma}_{(\boldsymbol{n}+1)} & \boldsymbol{\sigma}_{(\boldsymbol{n}+1)}^{\text {trial }} & K \Delta \varepsilon_{p} \mathbf{1} & 2 G \Delta \varepsilon_{q} \mathbf{n}_{(n+1)}^{\text {trial }}\end{array}$

with:

$\mathbf{n}_{(n+1)}^{\text {trial }} \quad\left(\frac{3 \boldsymbol{\sigma}^{\prime}}{2 \sigma_{e}}\right)_{(n+1)}^{\text {trial }}, \quad \boldsymbol{\sigma}_{(n+1)}^{\text {trial }} \quad \boldsymbol{\sigma}_{(n+1)}^{\text {trial }} \quad \sigma_{m(n+1)}^{\text {trial }} \mathbf{1}$,

$\sigma_{m(n+1)}^{\text {trial }} \quad \frac{1}{3} \boldsymbol{\sigma}_{(n+1)}^{\text {trial }}: \mathbf{1}, \quad$ and $\quad \sigma_{e(n+1)}^{\text {trial }} \quad \sqrt{\frac{3}{2}} \boldsymbol{\sigma}_{(n+1)}^{\text {trial }}: \boldsymbol{\sigma}_{(n+1)}^{\text {trial }}$

which allows one to obtain, separating Eq. (24) into its deviatoric and hydrostatic components, the following relations:

$\begin{array}{lll}\sigma_{m(n+1)} & \sigma_{m(n+1)}^{\text {trial }} & K \Delta \varepsilon_{p} \\ \sigma_{e(n+1)} & \sigma_{e(n+1)}^{t r i a l} & 3 G \Delta \varepsilon_{q}\end{array}$

From the integration of Eq. (14) we have:

$\sigma_{Y(n+1)}^{T} \Delta \varepsilon^{p} \quad \sigma_{(n+1)}: \Delta \varepsilon^{p} \quad\left(\sigma_{m} \Delta \varepsilon_{p}+\sigma_{e} \Delta \varepsilon_{q}\right)_{(n+1)}$ 
After some algebraic transformations on Eqs. (19), (23), (26), the three scalar non linear equations which should be solved to obtain $\Delta \varepsilon_{p}, \Delta \varepsilon_{q}$, and $\bar{\varepsilon}^{p}$ read:

(a) $\Phi\left(\sigma_{m}, \sigma_{e}, \sigma_{Y}^{T}\right) \leqslant 0$

(b) $\Delta \varepsilon_{p}\left(\frac{\partial \Phi}{\partial \sigma_{e}}\right) \quad \Delta \varepsilon_{q}\left(\frac{\partial \Phi}{\partial \sigma_{m}}\right) \quad 0$

(c) $\Delta \varepsilon^{p} \quad\left(\sigma_{m} \Delta \varepsilon_{p}+\sigma_{e} \Delta \varepsilon_{q}\right) / \sigma_{Y}^{T}$

with $\sigma_{Y}^{T} \quad \sigma_{Y}^{T}\left(\bar{\varepsilon}^{p}\right), \sigma_{m} \quad \sigma_{m}^{\text {trial }} \quad K \Delta \varepsilon_{p}$ and $\sigma_{e} \quad \sigma_{e}^{\text {trial }} \quad 3 G \Delta \varepsilon_{q}$, and all variables evaluated in $(n+1)$ and omitted for simplicity. The values of the variables are obtained following an iterative process using the Newton Raphson procedure, with the updated stress at time $(n+1)$ of the form:

$\boldsymbol{\sigma}_{(\boldsymbol{n}+1)} \quad \frac{2}{3} \sigma_{e(n+1)} \mathbf{n}_{(n+1)}^{\text {trial }}+\sigma_{m(n+1)} \mathbf{1}$

\section{Consistent tangent operator}

To preserve the quadratic rate of asymptotic convergence of the iterative solution based on Newton's method, a consistent tangent operator J, different in general from the continuum tangent moduli, is proposed by the enforcement of the consistency condition at the end of the step $(n+1)[26]$ :

$\mathbf{J}\left(\frac{\partial \boldsymbol{\sigma}}{\partial \boldsymbol{\varepsilon}}\right)_{(n+1)}\left(\frac{\partial \boldsymbol{\Delta} \boldsymbol{\sigma}}{\partial \boldsymbol{\Delta} \boldsymbol{\varepsilon}}\right)_{(n+1)}$

Deriving Eq. (10) and omitting the subscript $(n+1)$ for simplicity, it follows:

$\delta \boldsymbol{\Delta} \boldsymbol{\sigma} \quad\left(2 G \mathbf{I}^{\prime}+K \mathbf{1} \otimes \mathbf{1}\right): \delta \boldsymbol{\Delta} \boldsymbol{\varepsilon} \quad K \delta \Delta \varepsilon_{p} \mathbf{1} \quad 2 G \delta \Delta \varepsilon_{q} \mathbf{n} \quad 2 G \Delta \varepsilon_{q} \delta \mathbf{n}$

and from Eq. (25):

$\delta \sigma_{m} \quad \delta \sigma_{m}^{\text {trial }} \quad K \delta \Delta \varepsilon_{p} \quad \delta \sigma_{e} \quad \delta \sigma_{e}^{\text {trial }} \quad 3 G \delta \Delta \varepsilon_{q}$

Considering the above relations and deriving the yield function (27.a) the plastic flow (27.b) the evolution of $\bar{\varepsilon}^{p}$ (27.c) and after some algebraic transformations the following equations are ob tained as a function of $\delta \sigma_{m}^{\text {trial }}$ and $\delta \sigma_{e}^{\text {trial }}$ :

$$
\begin{array}{lc}
\delta \Delta \varepsilon_{p} & C_{11} \delta \sigma_{m}^{\text {trial }}+C_{12} \delta \sigma_{e}^{\text {trial }} \\
\delta \Delta \varepsilon_{q} & C_{21} \delta \sigma_{m}^{\text {trial }}+C_{22} \delta \sigma_{e}^{\text {trial }} \\
\delta \varepsilon^{p} & C_{31} \delta \sigma_{m}^{\text {trial }}+C_{32} \delta \sigma_{e}^{\text {trial }}
\end{array}
$$

with $C_{i j}$ coefficients known.

Bearing in mind the relations:

$$
\begin{array}{ll}
\delta \sigma_{m}^{\text {trial }} & K(\delta \boldsymbol{\Delta} \boldsymbol{\varepsilon}): \mathbf{1} \\
\delta \sigma_{e}^{\text {trial }} & \frac{3}{2 \sigma_{e}^{\text {trial }}} \boldsymbol{\sigma}^{\prime \text { trial }}: \boldsymbol{\delta} \boldsymbol{\sigma}^{\prime \text { trial }}
\end{array}
$$$$
\delta \mathbf{n} \delta \mathbf{n}^{\text {trial }} \frac{3}{2 \sigma_{e}^{\text {trial }}} \delta \boldsymbol{\sigma}^{\prime \text { trial }} \frac{3}{2\left(\sigma_{e}^{\text {trial }}\right)^{2}} \delta \sigma_{e}^{\text {trial }} \boldsymbol{\sigma}^{\text {trial }}
$$

the consistent tangent operator can be written as:

$$
\begin{aligned}
& \mathbf{J} \quad K\left(1 \quad K C_{11}\right) \mathbf{1} \otimes \mathbf{1}+\left(\begin{array}{ll}
2 G & \left.6 \frac{G^{2} \Delta \varepsilon_{q}}{\sigma_{e}^{\text {trial }}}\right) \mathbf{I}^{\prime}
\end{array}\right. \\
& +\frac{3 K G}{\sigma_{e}^{\text {trial }}} C_{12} \mathbf{1} \otimes \boldsymbol{\sigma}^{\prime}{ }^{\text {trial }}+\frac{3 K G}{\sigma_{e}^{\text {trial }}} C_{21} \boldsymbol{\sigma}^{\prime \text { trial }} \otimes \mathbf{1} \\
& +9 \frac{G^{2}}{\left(\sigma_{e}^{\text {trial }}\right)^{2}}\left(C_{22}+\frac{\Delta \varepsilon_{q}}{\sigma_{e}^{\text {trial }}}\right) \boldsymbol{\sigma}^{\prime \text { trial }} \otimes \boldsymbol{\sigma}^{\prime \text { trial }}
\end{aligned}
$$

It should be noted that no matrix inversion is necessary in the definition of this operator.
The proposed algorithm was implemented in the commercial Finite Element code ABAQUS/Standard [30] through a user subrou tine UMAT.

\section{Simulation of tests on notched tensile specimens of 2024 T3 aluminum alloy [6]}

In order to analyse the behaviour of the proposed model and the integration algorithm, different tensile tests performed experimen tally by Wilson [6] were simulated. The material considered is the 2024 T351 aluminum alloy. The true stress true strain material characteristc $\left(\begin{array}{ll}\sigma_{Y}^{T} & \varepsilon\end{array}\right)$ obtained from uniaxial tensile tests [6] follows the Ramberg Osgood power law hardening relationship:

$\frac{\varepsilon}{\varepsilon_{0}} \quad \frac{\sigma_{Y}^{T}}{\sigma_{0}^{T}}+\mu\left(\frac{\sigma_{Y}^{T}}{\sigma_{0}^{T}}\right)^{n}$

where $\sigma_{0}^{T}$ is the reference stress (taken as the $0.2 \%$ offset of the yield strength), $\varepsilon_{0} \quad \sigma_{0}^{T} / E$ is the reference strain, and $\mu$ and $n$ are the hardening coefficient and hardening exponent respectively. These properties, including the Young Modulus $E$ and Poisson's ratio $v$ constants, are listed in Table 1. A compression test were also con ducted by Wilson [6], and the resulting $\sigma_{0}^{c}$ is given in Table 1 .

The geometry and dimension details of the notched round bar specimens are given in Fig. 2, where the nominal diameter is $d=12.7 \mathrm{~mm}$ the neck diameter is $t_{n}=6.35 \mathrm{~mm}\left(d / t_{n}=0.5\right)$, and the notch flank angle of the tubes $\alpha$ is equal to $45^{\circ}$ in all cases. The chosen notch root radii have three different values of $r=0.127,0.254$ and $0.508 \mathrm{~mm}$.

The experimental tests were simulated with the Finite Element commercial code ABAQUS/Standard [30]. Due to the symmetry of the model, only a quarter of the specimen needs to be taken into account. The numerical analysis consists of 5700 axi symmetric 8 node elements with reduced integration, and with a refined mesh near the notched region. The finite element mesh and the detail of the mesh in the notched region for the case of $r=0.508 \mathrm{~mm}$ are shown in Fig. 3 .

Figs. 46 show the load displacement curves obtained in numerical situation using the Burzyński paraboloid model for dif ferent values of the strength differential factor $k: k=1,1.06,1.1$ and 1.2. These results are compared with the experimentally ob served behaviour of the specimen [6].

If the behaviour of the material at compression is the same as at tension, the Burzyński Torre paraboloid yield function, with the va lue of $k \quad \sigma_{0}^{C} / \sigma_{0}^{T} \quad 1$, leads to the Huber Mises yield criterion. In all of the analysed specimens, the response of the notched round bar for this value $k=1.0$ overshoots the real response of the material. The same behaviour was obtained by Wilson [6] for the Huber Mises yield function. Using the constant value of $k \quad \sigma_{0}^{C} / \sigma_{0}^{T} \quad 407.49 /$ 384.051 .06 (Table 1 ) during the simulation, gives the results which better predict the behaviour of the specimen and the simula tions match the experimental data when the chosen $k$ values are in the range of 1.06 and 1.1 .

\section{Simulation of tests on the Inconel 718 thin-walled tubes subjected to torsion after tension or compression [12]}

The strength differential effect has been also observed by Gil et al. [31,32] in the aged Inconel 718, a nickel base superalloy used

Table 1

Material properties for the 2024-T351 aluminum alloy at room temperature.

\begin{tabular}{llllll}
\hline$E(\mathrm{MPa})$ & $v$ & $\sigma_{0}^{T}(\mathrm{MPa})$ & $n$ & $\mu$ & $\sigma_{0}^{C}(\mathrm{MPa})$ \\
\hline 71,708 & 0.33 & 384.05 & 15 & 0.86 & 407.49 \\
\hline
\end{tabular}




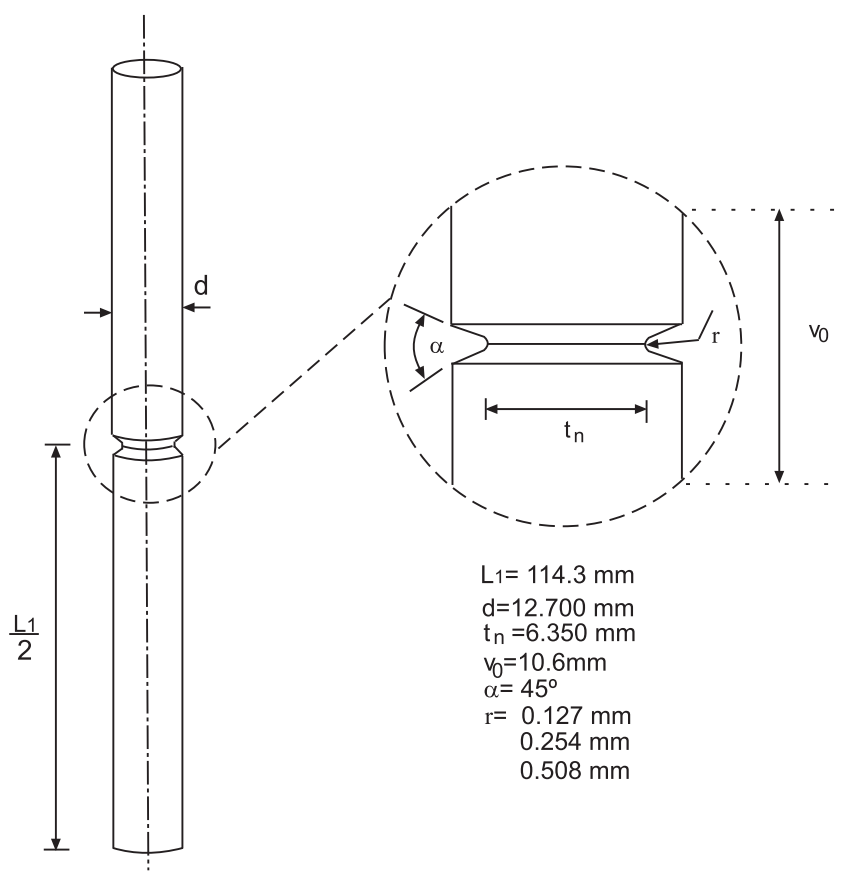

Fig. 2. Configuration of the notched specimen with an axisymmetric close up of the notch.

extensively for aeropropulsion structures. The elastic properties of the material are $E=165 \mathrm{GPa}$ and $v=0.297$, and the stress strain responses in uniaxial tension and compression reported by Iyer an Lissenden [12] are presented in Fig. 9. Iyer and Lissenden [12] proposed a set of experiments with non proportional loads to char acterize this material. The experiments developed by [12] consist in axial torsional tests of tubular specimens (subjected to an axial force and torque as shown in Fig. 7) with the outer and inner

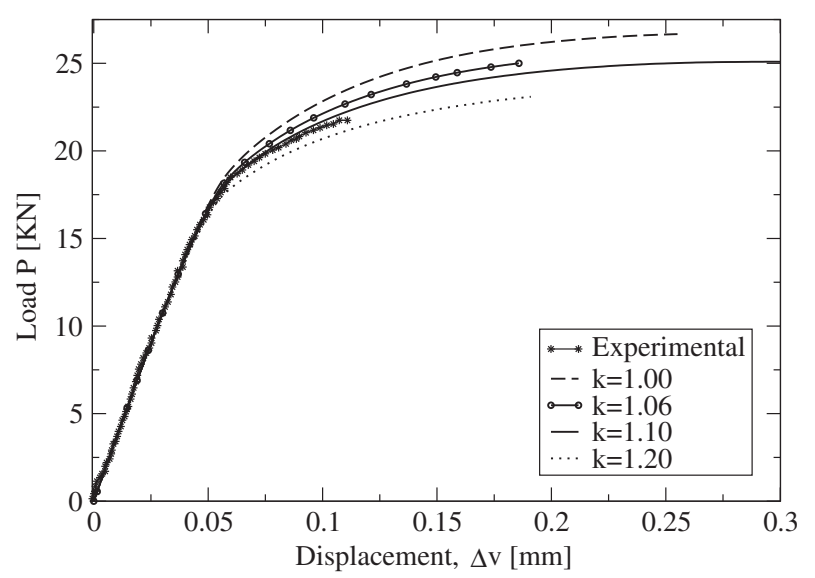

Fig. 4. Load-displacement results for $r=0.127 \mathrm{~mm}[6]$.

diameters of $D_{0}=21.0 \mathrm{~mm}$ and $D_{i}=15.9 \mathrm{~mm}$ respectively, and the length $L_{g}=25 \mathrm{~mm}$. The measured quantities considered were: axial displacement $(\delta)$, load $(P)$, torque $(T)$, and angle of twist $(\theta)$. From these quantities, the stresses and strains were calculated as:

$\sigma_{11} \frac{4 P}{\pi\left(D_{0}^{2} \quad D_{i}^{2}\right)} \quad \varepsilon_{11} \quad \frac{\delta}{L_{g}}$

for tension and compression tests, and

$\sigma_{12} \frac{16 T D_{0}}{\pi\left(D_{0}^{4} \quad D_{i}^{4}\right)} \quad \varepsilon_{12} \quad \frac{\gamma_{12}}{2} \quad \frac{D_{0} \theta}{2 L_{g}}$

for torsion tests. The path of biaxial loading applied to the speci mens and simulations consists in a uniaxial tension followed by tor sion (see Fig. 10a) and a uniaxial compresion followed by torsion (Fig. 10b). These tests were simulated by the FEM with the Burzyński Torre paraboloid model.
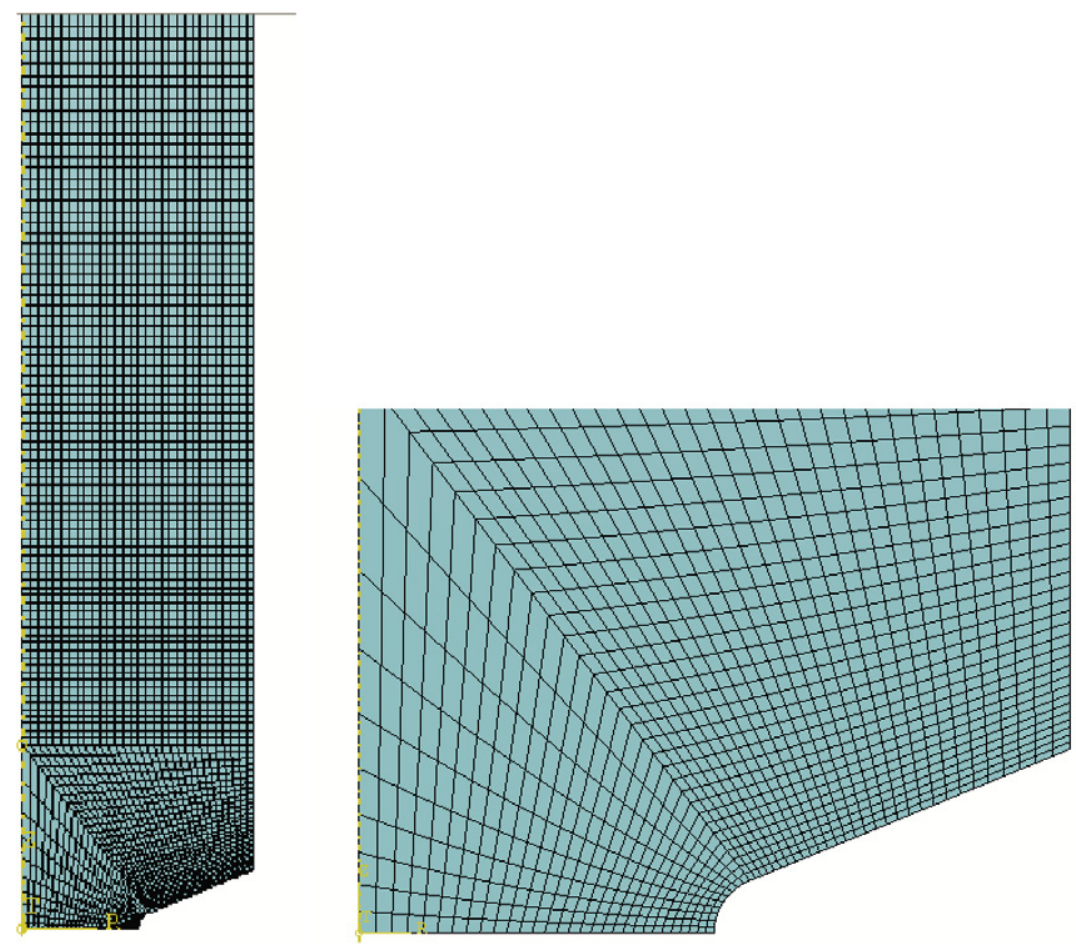

Fig. 3. The finite element mesh and the detail mesh near the noth region $(r=0.508 \mathrm{~mm})$. 


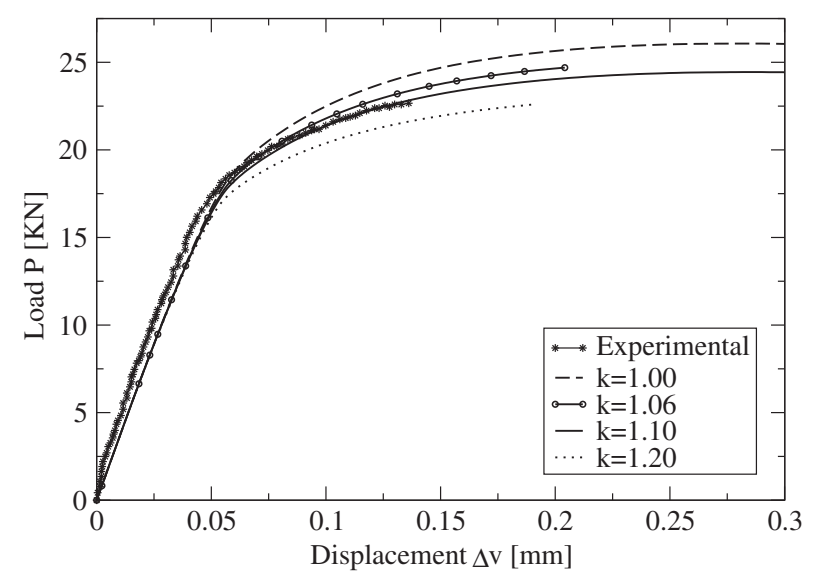

Fig. 5. Load-displacement results for $r=0.254 \mathrm{~mm}$ [6].

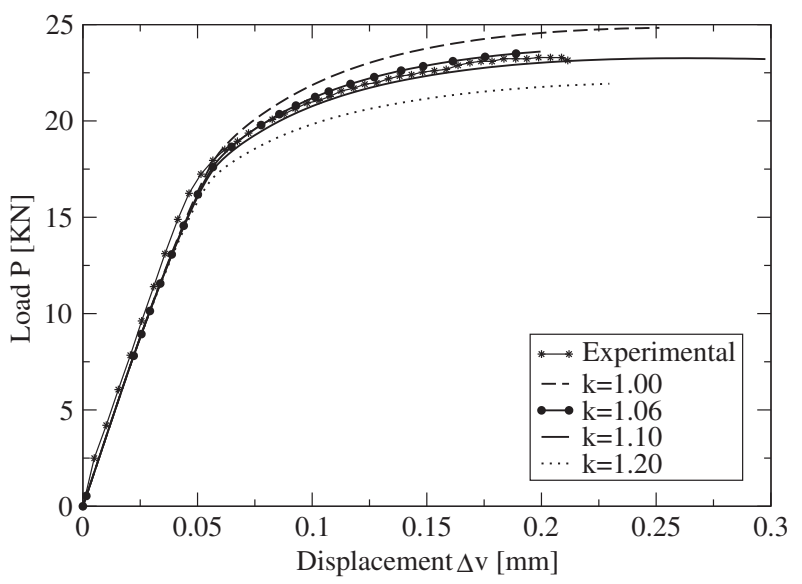

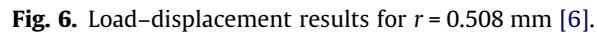

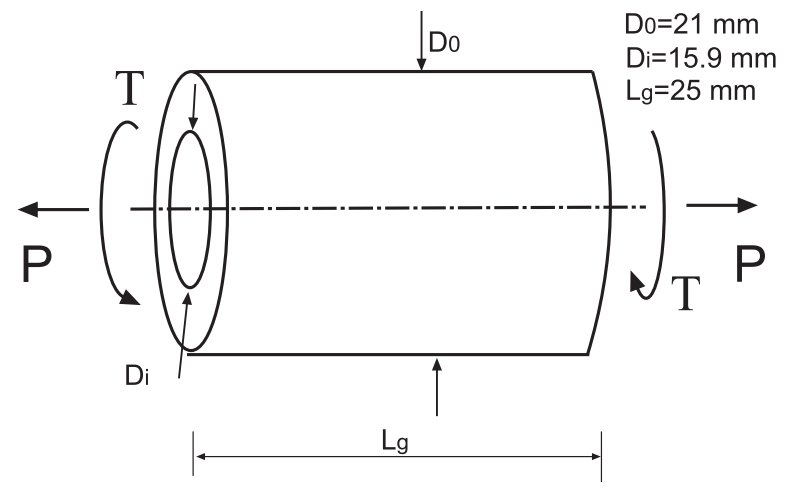

Fig. 7. Dimensions of the thin-walled tubes.

The finite element mesh used in the computations in ABAQUS/ Standard [30], with a total of 46008 node elements, is shown in Fig. 8, and the simulations of the specimen considering the non proportional load paths cases are compared with the experimental results given by [12].

Figs. 1114 present the comparison between experimental results and simulations for the Burzyński Torre paraboloid model with two different values of the strength differential factor: $\sigma_{Y}^{C} / \sigma_{Y}^{T} 1.0$ (where the strength differential is not considered)

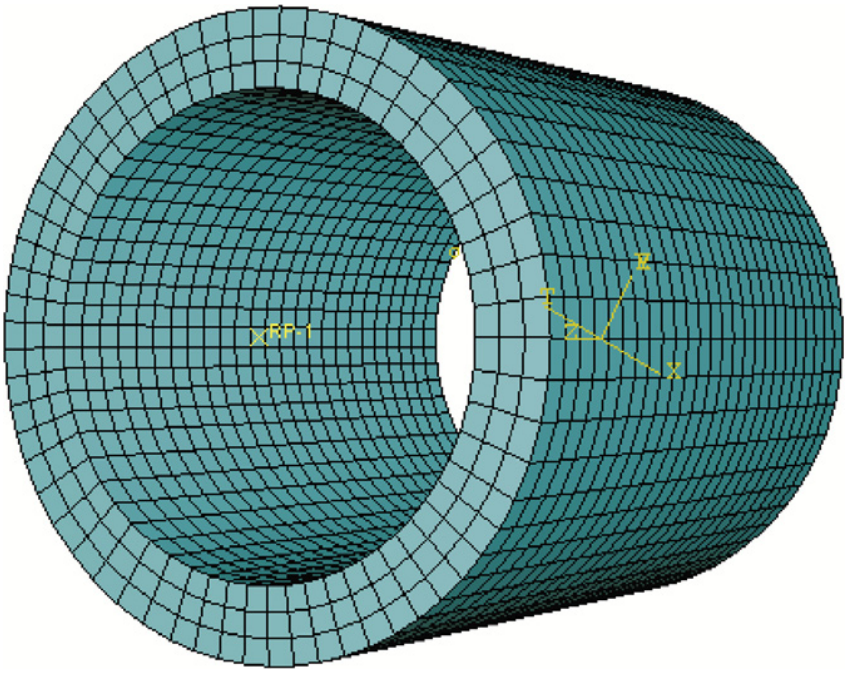

Fig. 8. Finite element mesh of thin walled tubes.

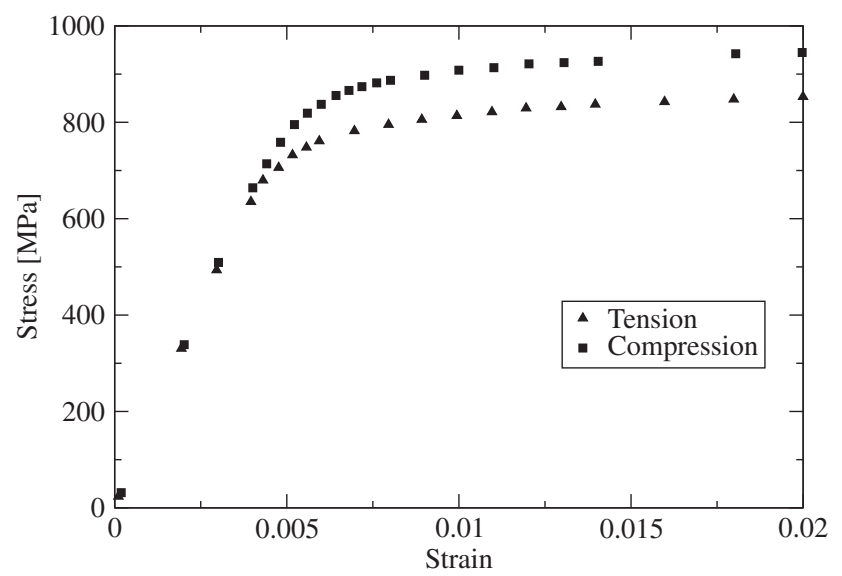

Fig. 9. Stress-strain curves for the Inconel 718 at tension and compression [12].

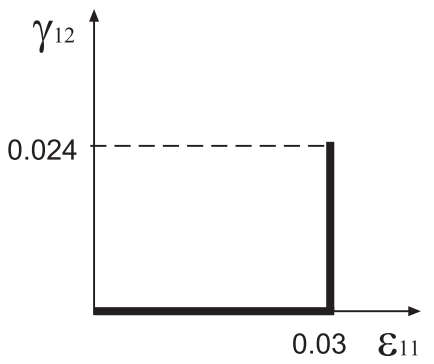

(a)

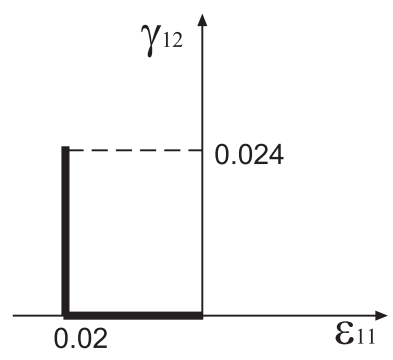

(b)
Fig. 10. Path of biaxial loading applied to the specimens: tension followed by torsion (a), compression followed by torsion (b).

and $k=1.1$ for two non proportional load paths: tension followed by torsion (Figs. 11 and 12) and compression followed by torsion (Figs. 13 and 14).

In Figs. 11 and 12, where the load path applied to the specimen consists in traction torsion tests following the displacement path (axial and radial) shown in Fig. 10a, the obtained curves for both $k$ values provide good agreement with experimental data.

However, in Figs. 13 and 14, a significant discrepancy is shown for the torsion after compression test (Fig. 10b) between the 


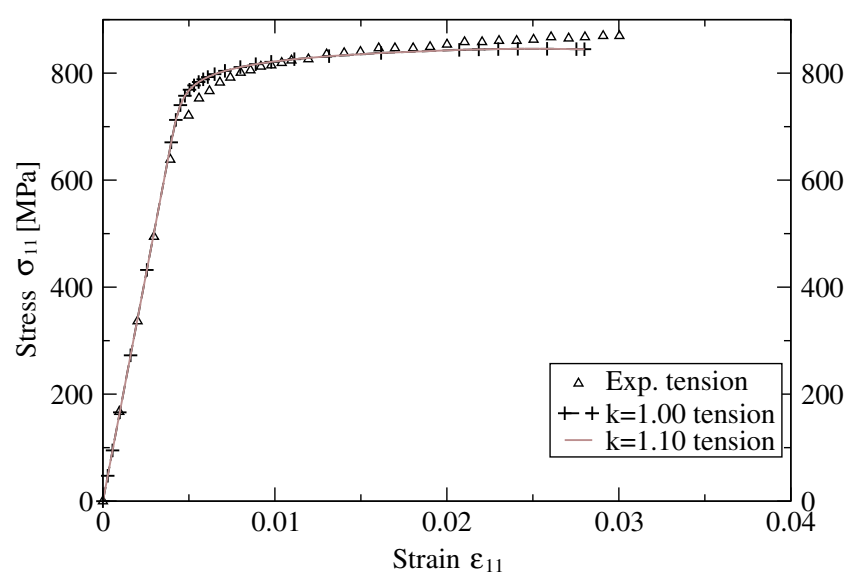

Fig. 11. Comparison between numerical and experimental results of tension followed by torsion tests: tension.

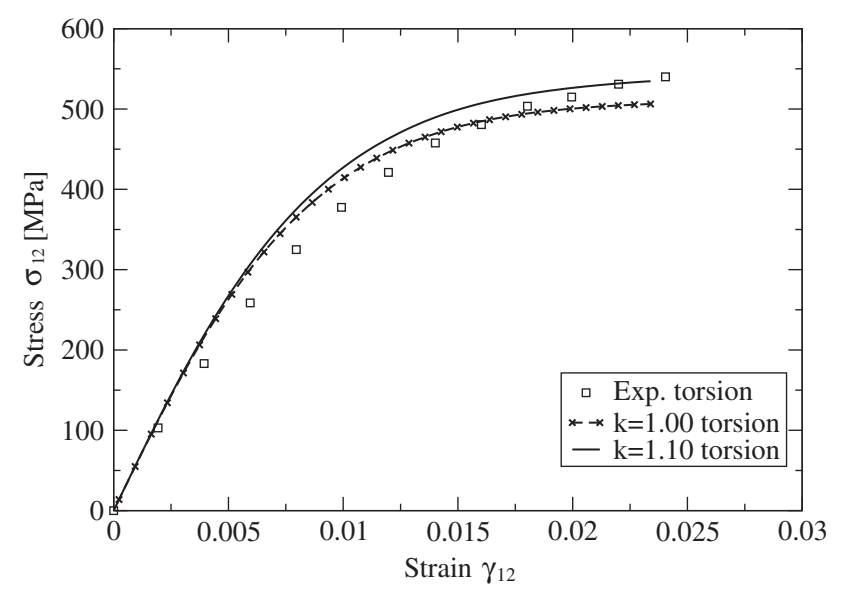

Fig. 12. Comparison between numerical and experimental results of tension followed by torsion tests: torsion after tension.

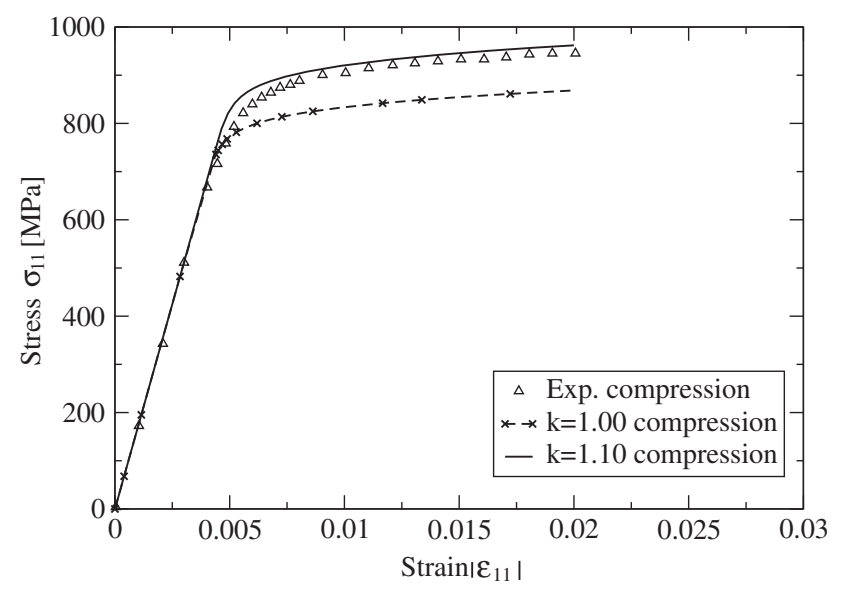

Fig. 13. Comparison between numerical and experimental results of compression followed by torsion tests: compression.

experimental data and the results of simulations where the Bur zyński model with $k=1.0$ is used. Note that this case corresponds with the consideration of the non SD effect. Nevertheless, for $k=1.1$ there is an excellent agreement between the numerical re sults and the experimental data, highlighting how important con

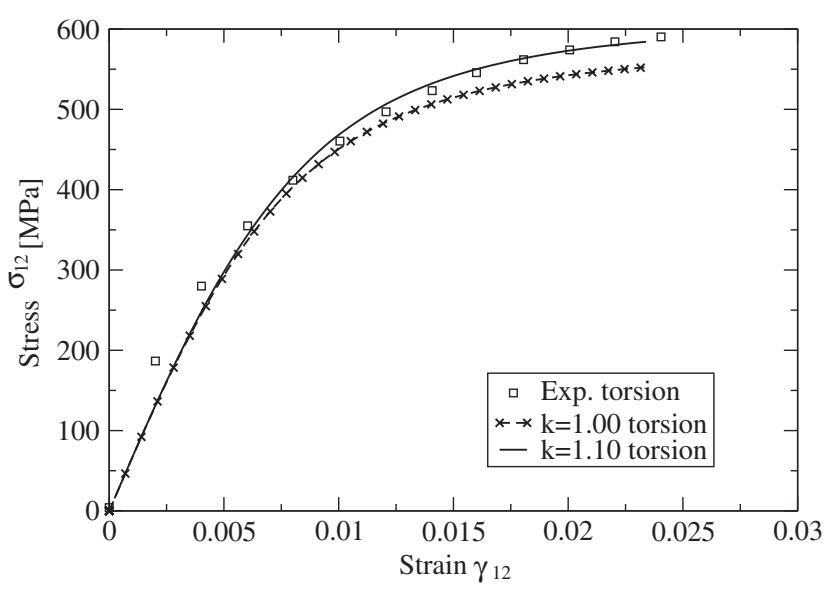

Fig. 14. Comparison between numerical and experimental results of compression followed by torsion tests: torsion after compression.

sidering of the strength differential effect for a correct simulation of the behaviour of the material at such a loading path is.

\section{Conclusions}

With the plasticity theory based on the Burzyński yield condi tion, we have simulated tensile tests on notched specimens of the 2024 T3 aluminum alloy [6], as well as tests on the thin walled tubes of Inconel 718 subjected to torsion after tension or compres sion [12]. The comparison between numerical and experimental results reveals that the application of the plasticity theory with the paraboloid case of the Burzyński yield condition correlates bet ter with experimental data than the results obtained with use of the classical plasticity theory with the Huber Mises yield condi tion. These observations can lead to more general conclusion that the discussed plasticity model with the paraboloid yield condition makes it possible to describe adequately the strength differential (SD) effect present also in other materials, as. e.g. high strength metals, in particular with nano grains, as well as, in polymers, for example in polycarbonates. The presented analysis creates also a possibility for the formulation of a similar integration scheme in the case of anisotropic materials for instance as in the methodol ogy developed by Oller et al. [33] which is based on the paraboloid isotropic yield criterion as a starting point. The criterion is to be ad justed by certain transformation to the behaviour of an orthotropic material.

\section{Acknowledgments}

The authors gratefully acknowledge the financial support given by the Spanish Ministerio de Educación y Ciencia, Project Reference DPI/2008 06408.

\section{References}

[1] Spitzig WA, Sober RJ, Richmond O. The effect of hydrostatic pressure on the deformation behavior of maraging and HY-80 steels and its implications for plasticity theory. Metall Trans A 1976;7A:1703-10.

[2] Richmond O, Spitzig WA. Pressure dependence and dilatancy of plastic flow. Int Union Theor Appl Mech Conf Proc 1980:377-86.

[3] Burzyński W. Ueber die Anstrengungshypothesen. Schweiz Bauzeitung 1929;94:259-62.

[4] Drucker DC, Prager W. Soil mechanics and plastic analysis of limit design. Quart J Appl Math 1952;10:157-65.

[5] Drucker DC. Plasticity theory, strength-differential (SD) phenomenon, and volume expansion in metals and plastics. Metall Trans 1973;4:667.

[6] Wilson CD. A critical reexamination of classical metal plasticity. J Appl Mech 2002;69:63-8. 
[7] Guo TF, Faleskog J, Shih CF. Continuum modeling of a porous solid with pressure-sensitive dilatant matrix. J Mech Phys Solids 2008;56:2188-212.

[8] Subramanya HY, Viswanath S, Narasimhan R. Influence of crack tip constraint on void growth in pressure sensitive plastic solids: I. 2D analysis. Eng Fract Mech 2008;75:1045-63.

[9] Theocaris PS. Failure criteria for isotropic bodies revisited. Eng Fract Mech 1995;51:239-64.

[10] Zhang H, Ramesh KT, Chin ESC. A multi-axial constitutive model for metal matrix composites. J Mech Phys Solids 2008;56:2972-83.

[11] Pẹcherski RB. Burzyński yield condition vis-à-vis the related studies reported in the literature. Eng Trans 2008;56:311-24.

[12] Iyer SK, Lissenden CJ. Multiaxial constitutive model accounting for the strength-differential in inconel 718. Int J Plasticity 2003;19:2055-81.

[13] Burzyński W. Theoretical foundations of the hypotheses of material effort. Eng Trans 2008;56:269-305. the recent edition of English translation of the paper in Polish published in 1929 in Czasopismo Techniczne, 47, 1-41.

[14] Nardin A, Zavarise G, Schrefler BA. Modelling of cutting tool soil interaction part I: contact behaviour; 2003.

[15] Kolupaev VA, Kolling S, Bolchoun A, Moneke M. A limit surface formulation for plastically compressible polymers. Mech Compos Mater 2007;43:245-58.

[16] Kordzikowski P, Janus-Michalska M, Pęcherski RB. Specification of energybased criterion of elastic limit states for cellular materials. Arch Metall Mater 2005;50:621-34.

[17] Kolupaev VA, Kraatz A, Moneke M, Bolchoun A. Beschreibung der mehraxialen kriechphSnomene bei hartschaumstoffen, KGK Kautschuk Gummi Kunststoffe 59 (2006) 17-26.

[18] Zyczkowski M. Combined loadings in the theory of plasticity. Warszawa: PWN-Polish Scientific Publishers; 1981.

[19] Zyczkowski M. Discontinuous bifurcations in the case of the Burzyński-Torre yield condition. Acta Mech 1999;132:19-35.
[20] Skrzypek JJ. Plasticity and creep theory, examples, and problems. Boca Raton: R.B. Hetnarski, CRC Press; 1993.

[21] Jirásek M, Bazant ZP. Inelastic analysis of structures. Chichester: J. Wiley; 2002.

[22] Huber MT. Właściwa praca odkształcenia jako miara wytęzenia materjału, czasopismo techniczne. Lwów; 1904 [see also the recent edition of English translation in the centennial of original Polish publication: specific work of strain as a measure of material effort. Arch Mech 2004;56:173-90].

[23] Mises RV. Mechanik der festen Körper im plastisch-deformablen Zustand, Göttingen Nachrichten. Math Phys Klasse Z 1913;4 (1): 582-92.

[24] Hencky H. Zur theorie plastischer deformationen und der hierdurch im material hervorgerufenen nachspannungen. ZAMM 1924;4:323-34.

[25] Torre C. Grenzbedingungen für spröden Bruch und plastisches Verhalden bildsamer. Metall Ing Archiv 1950;4:174-89.

[26] Simo JC, Taylor RL. Consistent tangent operators for rate-independent elastoplasticity. Comput Methods Appl Mech Eng 1985;48:101-18.

[27] Simo JC, Hughes T. Computational inelasticity. New York: Springer; 1998.

[28] Aravas N. On the numerical integration of a class of pressure-dependent plasticity models. Int J Numer Methods Eng 1987;24:1395-416.

[29] Vadillo G, Zaera R, Fernández-Sáez J. Consistent integration of the constitutive equations of Gurson materials under adiabatic conditions. Comput Methods Appl Mech Eng 2008;197:1280-95.

[30] HKS, ABAQUS/Standard v6.8 User's Manual. Richmond (USA): ABAQUS Inc.; 2009.

[31] Gil CM, Lissenden CJ, Lerch BA. Yield of Inconel 718 by axial-torsional loading at temperatures up to 649c. J Test Eval 1999;27:327.

[32] Gil CM, Lissenden CJ, Lerch BA. Unusual nonlinear response of some metallic materials. Mech Mater 1999;31:565.

[33] Oller S, Car E, Lubliner J. Definition of a general implicit orthotropic yield criteria. Comput Methods Appl Mech Eng 2003;192:895-912. 